\title{
Balloon aortic valvuloplasty, Impella insertion and complex coronary intervention: is this all feasible fully percutaneously via upper limb access?
}

\author{
Jerzy Sacha ${ }^{1,2}$, Krzysztof Krawczyk², Maciej Gawor³, Joanna Płonka², Marek Gierlotka² \\ ${ }^{1}$ Faculty of Physical Education and Physiotherapy, Opole University of Technology, Opole, Poland \\ ${ }^{2}$ Department of Cardiology, University Hospital, Institute of Medical Sciences, University of Opole, Opole, Poland \\ ${ }^{3}$ Department of Anesthesiology and Intensive Care, University Hospital, Institute of Medical Sciences, University of Opole, Opole, Poland
}

Adv Interv Cardiol 2021; 17, 1 (63): 126-128

DOI: https://doi.org/10.5114/aic.2021.104783

Complex high-risk indicated patients (CHIP) with a limited vascular access constitute a real challenge for percutaneous coronary interventions $(\mathrm{PCl})$, particularly if they require mechanical circulatory support devices. A 73-year-old man with recent non-ST segment elevation myocardial infraction, depressed left ventricular function (ejection fraction, 40\%), aortic stenosis (max/mean gradient, $50 / 32 \mathrm{~mm} \mathrm{Hg}$; aortic valve area and its index, $1.0 \mathrm{~cm}^{2}$ and $0.5 \mathrm{~cm}^{2} / \mathrm{m}^{2}$ ) and numerous comorbidities (including recently diagnosed lung cancer in the initial phase) was scheduled by the heart team for balloon aortic valvuloplasty (BAV) and Impella-supported complex $\mathrm{PCl}$ of the left main (LM) and the left anterior descending artery (LAD) (Figure $1 \mathrm{~A}$ ) - the dominant right coronary artery was chronically occluded without the viability of the corresponding myocardium. Due to the abdominal aortic aneurysm with intraluminal thrombus, the procedure could only be performed via upper limb access, and the following interventional images demonstrate how to do it fully percutaneously without general anesthesia.

Through the left radial artery, a safety guidewire was inserted into the aorta and the $10 / 40 \mathrm{~mm}$ peripheral balloon was placed within the left axillary artery just below the clavicula, i.e. the access site for the Impella insertion. Under ultrasound imaging and fluoroscopy, the axillary artery and the balloon were punctured (this technique prevents puncturing the opposite arterial wall and the vessel dissection); the access-site guidewire was inserted into the balloon, and then they were both pushed into the aorta (Figure 1 B) [1, 2]. The balloon was replaced with a new one that served as a hemostatic tamponade during maneuvers within the access site. After placing Proglides and the 14F Impella sheath, a pre-shaped stiff guidewire was inserted into the left ventricle, which was used as an electrode for rapid pacing during BAV with a 20/40 $\mathrm{mm}$ balloon (Figure $1 \mathrm{C}$ ). Then, the Impella CP was implanted via the transaxillary access and $\mathrm{PCl}$ of the $L M$ and $L A D$ was performed through the right radial artery (Figure $1 \mathrm{D}$ ). After the procedure, the Impella and its sheath were removed during the inflation of the peripheral balloon delivered over the safety guidewire (Figure $1 \mathrm{E}$ ) - such a technique prevents bleeding during closing the access site. Finally, the access site was successfully closed percutaneously with Proglides. It should be noted that the safety and access-site guidewires were kept in place until the angiography confirmed a good hemostatic effect (Figure $1 \mathrm{~F}$ ). In case of vascular complications, the safety guidewire may serve for implantation of stents or covered stents, but the access-site guidewire may be used for additional Proglides or Angio-Seal delivery - such a technique makes the axillary approach quite safe [1-3]. Several days later the patient was discharged home in a good condition.

\section{Acknowledgments}

We thank Ewa Podżus, Małgorzata Demkowicz, Małgorzata Buchta and Dagmara Danicka for their assistance during the operation.

\section{Conflict of interest}

The authors declare no conflict of interest.

\section{Corresponding author:}

Jerzy Sacha MD, PhD, Faculty of Physical Education and Physiotherapy, Opole University of Technology, 76 Prószkowska St, $45-758$ Opole, Poland, phone: +487745208 72, e-mail: sacha@op.pl

Received: 27.10.2020, accepted: 26.11.2020. 

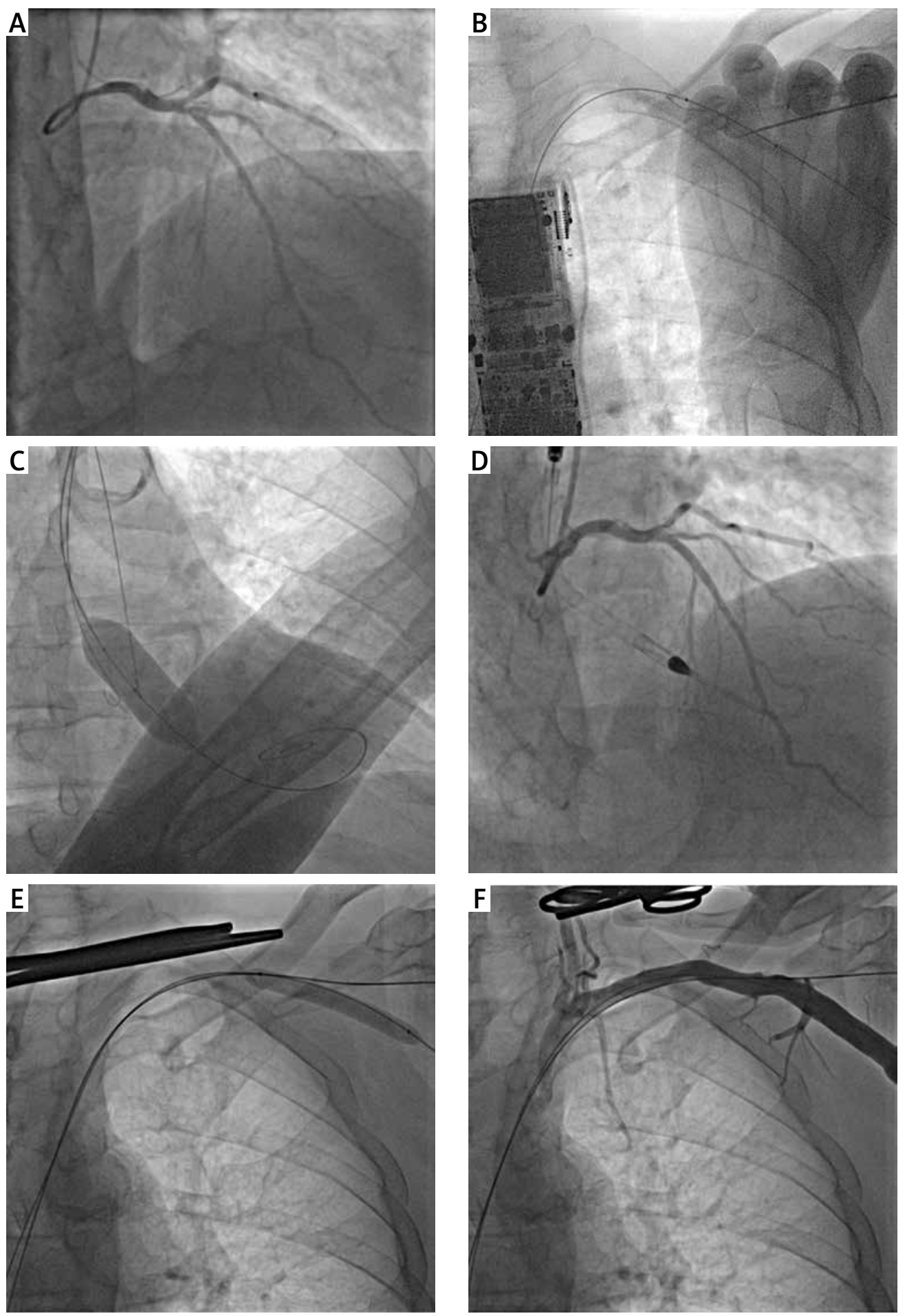

Figure 1. Interventions via upper limb access. A - Coronary angiography shows the ostial left main and complex left anterior descending artery stenoses - the circumflex artery is rather small. B - The axillary artery was punctured on the fully inflated balloon, then the access-site guidewire was inserted into the balloon, and they were both delivered to the aorta. C - Balloon aortic valvuloplasty was performed (the pre-shaped stiff guidewire was used for rapid pacing). D - The Impella CP was inserted via the left axillary access, but percutaneous coronary intervention was performed via the right radial access with a good final result. $E$ - Balloon tamponade of the access site was employed to prevent torrential bleeding during the removal of the Impella sheath. $\mathbf{F}$ - The angiography confirms the successful percutaneous closure of the access site. One can see two guidewires for a possible bailout procedure, i.e.: the access-site guidewire enables an additional placement of Proglides or Angio-Seal, while the safety guidewire may serve for implantation of stents or covered stents in case of vascular complications 


\section{References}

1. Sacha J, Krawczyk K, Gierlotka M. Fully percutaneous insertion and removal of the Impella CP via a subclavian approach. Adv Interv Cardiol 2020; 16: 343-6.

2. Sacha J, Krawczyk K, Gwóźdź W, et al. Fully percutaneous transaxillary aortic valve replacement with effective bailout plan for vascular complications. JACC Cardiovasc Interv 2020; 13: 2811-2.

3. Schäfer U, Ho Y, Frerker C, et al. Direct percutaneous access technique for transaxillary transcatheter aortic valve implantation: "the Hamburg Sankt Georg approach". JACC Cardiovasc Interv 2012; 5: 477-86. 